\title{
Unilateral Nasal Obstruction Causes Symptom Severity Scores Similar to Bilateral Nasal Obstruction
}

\author{
Rachel E. Weitzman, MS ${ }^{1}$ Allen L. Feng, MD ${ }^{1} \quad$ Natalie Justicz, MD ${ }^{1} \quad$ Shekhar K. Gadkaree, MD ${ }^{1}$ \\ Robin W. Lindsay, MD ${ }^{1}$ \\ 1 Department of Otolaryngology, Mass Eye and Ear Main Campus, \\ Boston, Massachusetts \\ Facial Plast Surg 2020;36:487-492.

\begin{abstract}
Address for correspondence Rachel E. Weitzman, MS, Department of Otolaryngology, Harvard Medical School, 25 Shattuck Street, Boston, MA 02115 (e-mail: rachel_weitzman@hms.harvard.edu; weitzmanre@gmail.com).
\end{abstract}

\begin{abstract}
Keywords

- nasal obstruction

- septorhinoplasty

- NOSE scale

- nasal anatomic worksheet

The objective of this paper is to investigate the differences between unilateral and bilateral nasal obstruction with subjective symptomatology in nasal obstruction. This is a retrospective chart review of prospectively collected data of patients with nasal obstruction presenting to a facial plastic and reconstructive surgery clinic for evaluation and treatment. Patient demographics including age and gender were recorded along with nasal physical exam findings. These included internal valve narrowing (IVN), external valve narrowing, internal valve collapse, external valve collapse, and septal deviations (inferior and superior). Findings were reported for the left, the right, and both sides. Nasal valve and septal findings were recorded on 3- and 4-point Likert scales, respectively, for each side of the nose. A total of 1,646 patients were included in the study. On univariate analysis, a significant correlation was seen between Nasal Obstruction Symptom Evaluation (NOSE) scores and all individual exam findings $(p<0.001)$. On multiple linear regression, total, left, and right septal deviation $(p<0.001, p=0.001, p=0.007$, respectively) and total, left, and right IVN $(p<0.001, p=0.003, p<0.001)$ were all predictive of an increased NOSE score. Patients with unilateral septal deviation or internal nasal valve narrowing have symptoms of nasal obstruction similar to those with bilateral nasal obstruction. Unilateral and bilateral septal deviation and internal nasal valve narrowing are predictive of having an increased NOSE score. Unilateral nasal obstruction should be recognized and treated as a cause for severe symptomatic nasal obstruction despite a normal contralateral nasal exam.
\end{abstract}

Despite the growing wealth of research on the negative impact of nasal obstruction on patient quality of life ( $Q o L)$, little is understood about the impact of unilateral nasal obstruction. As objective techniques to measure nasal airflow and resistance are developed for clinical use, it will be increasingly important to understand the relationship between airflow and subjective nasal symptomatology. The symptomatic patient with unilateral nasal obstruction on physical examination allows an opportunity to examine this relationship.

Previous research efforts have demonstrated that nasal airway obstruction (NAO) represents a common problem in otolaryngology, affecting approximately 20 million Americans with a significant impact on QoL. ${ }^{1-4}$ Nasal obstruction can negatively impact both disease-specific and global QoL, as well as, negatively impact sleep. Medical management for NAO ranges from topical decongestant and steroid nasal sprays to oral allergy medications to nasal strips. Surgical management is traditionally more variable and individualized and may include septoplasty, turbinoplasty, nasal valve correction, or functional septorhinoplasty.

Valid patient-reported outcomes measures are crucial to providing insight into the impact of NAO. ${ }^{5}$ The Nasal Obstruction Symptom Evaluation (NOSE) survey is a validated QoL instrument for patients with nasal obstruction, commonly published online July 27,2020
Issue Theme Controversies in Facelifting; Guest Editor: Fred G. Fedok, MD, FACS
Copyright $\odot 2020$ by Thieme Medical Publishers, Inc., 333 Seventh Avenue, New York, NY 10001, USA. Tel: +1(212) 760-0888.
DOI https://doi.org/ 10.1055/s-0040-1714265. ISSN $0736-6825$. 
used to assess outcomes following septoplasty and rhinoplasty. ${ }^{6}$ We have previously demonstrated significant improvements in the NOSE score after functional rhinoplasty, and revealed that history of septoplasty, snoring, and internal valve narrowing (IVN) are associated with increased NOSE scores in patients presenting preoperatively for functional septorhinoplasty. ${ }^{6-8}$ However, the impact of unilateral nasal obstruction on symptom severity has not been thoroughly evaluated.

In this study, we utilized systematic physical examination reporting to study the relationship of unilateral nasal characteristics on NOSE score severity. We find that left or right (as well as total) internal nasal valve narrowing and septal deviation independently predict NOSE score severity. These data demonstrate that patients can present with complaints of severe symptomatic nasal obstruction even when the physical examination demonstrates only unilateral nasal abnormalities.

\section{Methods}

\section{Patient Selection}

A retrospective chart review was performed at a tertiary care medical center under an approved protocol by the institutional review board human subjects research committee. The study period spanned 6 years (2012-2018). Eligible subjects involved adult and pediatric (less than 18 years old) patients who presented to the Massachusetts Eye and Ear Infirmary Facial Plastic and Reconstructive Surgery (Boston, MA) clinic for assessment of NAO by the senior author (R.W.L.). The subjects were referred due to concern for NAO to discuss medical and surgical options, or for surgical intervention. The subjects completed a preoperative NOSE survey in the clinic electronically and underwent a standardized nasal history and physical exam. ${ }^{9}$ All data were collected in REDCap (Research Electronic Data Capture; Vanderbilt University, Nashville, TN), an electronic data-capture platform designed for academic clinical and translational database development. ${ }^{10}$ Patient demographic characteristics, nasal history, nasal exam, and preoperative NOSE scores were recorded through REDCap in a Health Insurance Portability and Accountability Act-compliant manner.

\section{Outcome Measures}

The NOSE scale was considered the primary outcome measure. The NOSE scale is a validated, patient-reported, diseasespecific QOL assessment instrument that contains five questions related to nasal obstruction rated along a 5-point Likert scale. ${ }^{6,11-13}$ Question response scores are summed and converted to a total score from 0 (no nasal obstruction) to 100 (severe nasal obstruction).

\section{Physical Exam}

Physical exam findings were recorded on a nasal exam and Nasal Anatomic Worksheet (NAW) in REDCap. The nasal exam worksheet focused on external nasal anatomy and the NAW on intranasal anatomy. ${ }^{9}$ On the NAW, deviation of the septum and nasal valve narrowing and collapse were analyzed by unique categories: left superior septal deviation, right superior septal deviation, left inferior septal deviation, and right inferior septal deviation on a 4-point Likert scale (rated 0 if not present, 1 for mild, 2 for moderate, 3 for severe), and left IVN at rest, right IVN at rest, left external valve narrowing (EVN) at rest, right EVN at rest, left internal valve collapse (IVC) with inspiration, right IVC with inspiration, left external valve collapse (EVC) with inspiration, and right EVC with inspiration on a 3-point Likert scale (rated 1 for absent/mild, 2 for moderate, 3 for severe). Each item was scored individually. The individual scores were combined to create the total NAW score.

\section{Statistical Analysis}

Univariate analysis was performed using one-way analysis of variance (ANOVA) tests to determine the association between interval variables and NOSE scores. Multivariable linear regression for NOSE scores was performed using a backward stepwise approach. Variance inflation factors (VIF) were calculated to determine multicollinearity ( $\mathrm{VIF}<4$ ). Data were analyzed using STATA 13.0 (StataCorp) with significance attributed to $p$-values $<0.05$.

\section{Results}

\section{Patient Characteristics}

Retrospective chart review identified 1,081 patients who presented to the Massachusetts Eye and Ear Infirmary Facial Plastic and Reconstructive Surgery clinic for evaluation of NAO (-Table 1). In total, $53.9 \%$ of patients were females, while $46.1 \%$ of patients were males. The mean patient age was $38.0 \pm 16.5$ years. The mean NOSE score was $59.7 \pm 25.0$.

\section{Physical Exam}

In the univariate analyses, the variables of left-sided EVN $(p=0.011)$, right-sided EVN $(p<0.001)$, left-sided EVC $(p<0.001)$, right-sided EVC $(p<0.001)$, left-sided IVN $(p<0.001)$, right-sided IVN $(p<0.001)$, left-sided IVC $(p<0.001)$, right-sided IVC $(p<0.001)$, left-sided inferior septal deviation $(p<0.001)$, right-sided inferior septal deviation $(p<0.001)$, left-sided superior septal deviation $(p<0.001)$, and right-sided superior septal deviation $(p<0.001)$ were significantly correlated with NOSE scores (-Table $\mathbf{2}$ ).

Table 1 Demographic values for patients with nasal airway obstruction

\begin{tabular}{|l|c|}
\hline Patient characteristics $(\boldsymbol{n}=\mathbf{1 , 0 8 1})$ & Value \\
\hline Sex $(n=1,075)$ & \\
\hline Female $(\%)$ & 53.9 \\
\hline Male $(\%)$ & 46.1 \\
\hline Age $(n=1,015)$ & \\
\hline Mean (y) & 38.0 \\
\hline Standard deviation $(\mathrm{y})$ & 16.5 \\
\hline NOSE $(n=1,081)$ & \\
\hline Mean & 59.7 \\
\hline Standard deviation & 25.0 \\
\hline
\end{tabular}

Abbreviation: NOSE, Nasal Obstruction Symptom Evaluation. 
Table 2 Physical exam results stratified by NOSE score with corresponding univariate analysis

\begin{tabular}{|c|c|c|c|c|}
\hline \multirow[t]{2}{*}{ Physical exam } & \multicolumn{2}{|c|}{ Left side } & \multicolumn{2}{|c|}{ Right side } \\
\hline & $\begin{array}{l}\text { NOSE score } \\
\text { (SD) }\end{array}$ & $p$-Value & $\begin{array}{l}\text { NOSE score } \\
\text { (SD) }\end{array}$ & $p$-Value \\
\hline \multicolumn{5}{|c|}{ External valve narrowing } \\
\hline 1 & $57.9(1.0)$ & \multirow[t]{3}{*}{0.011} & $57.1(1.0)$ & \multirow[t]{3}{*}{$<0.001$} \\
\hline 2 & $62.4(1.3)$ & & $64.8(1.3)$ & \\
\hline 3 & $62.9(2.0)$ & & $63.4(2.2)$ & \\
\hline \multicolumn{5}{|c|}{ External valve collapse } \\
\hline 1 & $57.4(0.9)$ & \multirow[t]{3}{*}{$<0.001$} & $57.4(0.9)$ & \multirow[t]{3}{*}{$<0.001$} \\
\hline 2 & $64.9(1.3)$ & & $65.5(1.3)$ & \\
\hline 3 & $66.5(2.9)$ & & $65.1(2.6)$ & \\
\hline \multicolumn{5}{|c|}{ Internal valve narrowing } \\
\hline 1 & $50.1(1.8)$ & \multirow[t]{3}{*}{$<0.001$} & $50.3(1.7)$ & \multirow[t]{3}{*}{$<0.001$} \\
\hline 2 & $62.1(1.2)$ & & $61.9(1.2)$ & \\
\hline 3 & $63.0(1.1)$ & & $64.0(1.1)$ & \\
\hline \multicolumn{5}{|c|}{ Internal valve collapse } \\
\hline 1 & $56.9(1.0)$ & \multirow[t]{3}{*}{$<0.001$} & $56.5(1.0)$ & \multirow[t]{3}{*}{$<0.001$} \\
\hline 2 & $65.7(1.3)$ & & $67.0(1.2)$ & \\
\hline 3 & $63.6(2.7)$ & & $62.7(2.3)$ & \\
\hline \multicolumn{5}{|c|}{ Inferior septal deviation } \\
\hline 0 & $54.9(1.3)$ & \multirow[t]{4}{*}{$<0.001$} & $56.5(1.2)$ & \multirow[t]{4}{*}{$<0.001$} \\
\hline 1 & $64.0(1.8)$ & & $62.4(1.7)$ & \\
\hline 2 & $61.7(1.6)$ & & $63.6(1.5)$ & \\
\hline 3 & $64.3(1.4)$ & & $63.8(1.7)$ & \\
\hline \multicolumn{5}{|c|}{ Superior septal deviation } \\
\hline 0 & $55.1(1.4)$ & \multirow[t]{4}{*}{$<0.001$} & $55.4(1.3)$ & \multirow[t]{4}{*}{$<0.001$} \\
\hline 1 & $63.6(1.8)$ & & $62.1(1.7)$ & \\
\hline 2 & $61.4(1.7)$ & & $62.4(1.6)$ & \\
\hline 3 & $63.2(1.2)$ & & $64.7(1.3)$ & \\
\hline
\end{tabular}

Abbreviation: NOSE, Nasal Obstruction Symptom Evaluation; SD, standard deviation.

In the multivariate analyses, inferior and superior septal deviations were found to be collinear and were taken instead as a total septal score. The total septal score and other nasal exam findings on each side of the nose were found to be predictive of an increased NOSE score. On the left-hand side, IVN score $(p=0.003)$ and total septum score $(p=0.001)$ were found to be significant predictors of an elevated NOSE score. On the right-hand side, similar results were seen with IVN score $(p<0.001)$ and total septum score $(p=0.007)$ being significant predictors of an elevated NOSE score. When looking at combined (bilateral) physical exam findings, similar results are shown with IVN score $(p<0.001)$ and total septum score $(p<0.001)$ being significant predictors. All regression variables for unilateral or bilateral analyses demonstrated VIF $\leq 3$. When considering unilateral or bilateral physical exam findings, IVC, EVN, and EVC were not predictive of an elevated NOSE score. Age and sex were similarly not associated with NOSE scores (- Table $\mathbf{3}$ ).

\section{Discussion}

Unilateral nasal obstruction is an incompletely understood problem. Clinical experience suggests that unilateral obstruction can create bothersome symptoms, but quantitative evidence of this phenomenon is lacking. Interestingly, the effect of unilateral nasal obstruction was investigated in snoring and sleep outcomes in the 1990s where unilateral nasal obstruction was found to impact snoring and sleep apnea as strongly as bilateral obstruction. ${ }^{14}$ Experiments in murine models of taste bud acquisition have also noted dramatic abnormalities of papillae development in rats subjected to unilateral nasal obstruction, demonstrating the importance of bilateral airflow for normal physiologic functions. ${ }^{15}$ In this study, we attempted to study the independent effect of unilateral nasal obstruction on the overall subjective symptom of nasal obstruction using the NOSE score and a standardized physical exam. We found that lateralizing internal nasal valve narrowing and septal 
Table 3 Multiple linear regressions for NOSE scores based on bilateral and unilateral physical exam findings

\begin{tabular}{|c|c|c|c|}
\hline NOSE score & Coefficient & $95 \% \mathrm{Cl}$ & $p$-Value \\
\hline \multicolumn{4}{|l|}{ Bilateral scores } \\
\hline IVN score & 3.133 & 1.783 to 4.482 & $<0.001$ \\
\hline IVC score & 1.962 & -0.426 to 4.350 & 0.107 \\
\hline EVN score & -0.238 & -1.705 to 1.230 & 0.751 \\
\hline EVC score & 1.145 & -1.403 to 3.693 & 0.378 \\
\hline Total septum score & 0.803 & 0.371 to 1.235 & $<0.001$ \\
\hline Age & 0.073 & -0.020 to 0.167 & 0.123 \\
\hline \multicolumn{4}{|l|}{ Sex } \\
\hline Male & Ref & & \\
\hline Female & 1.072 & -1.962 to 4.106 & 0.488 \\
\hline \multicolumn{4}{|l|}{ Left-sided scores } \\
\hline IVN score & 3.340 & 1.122 to 5.558 & 0.003 \\
\hline IVC score & 3.646 & -0.722 to 8.014 & 0.102 \\
\hline EVN score & -0.499 & -2.968 to 1.969 & 0.692 \\
\hline EVC score & 2.968 & -1.590 to 7.525 & 0.202 \\
\hline Total septum score & 1.346 & 0.570 to 2.122 & 0.001 \\
\hline Age & 0.072 & -0.022 to 0.166 & 0.134 \\
\hline \multicolumn{4}{|l|}{ Sex } \\
\hline Male & Ref & & \\
\hline Female & 0.817 & -2.245 to 3.879 & 0.601 \\
\hline \multicolumn{4}{|l|}{ Right-sided scores } \\
\hline IVN score & 4.230 & 1.991 to 6.470 & $<0.001$ \\
\hline IVC score & 4.077 & -0.271 to 8.424 & 0.066 \\
\hline EVN score & 1.059 & -1.646 to 3.763 & 0.443 \\
\hline EVC score & 1.570 & -3.132 to 6.271 & 0.513 \\
\hline Total septum score & 1.073 & 0.290 to 1.855 & 0.007 \\
\hline Age & 0.050 & -0.043 to 0.143 & 0.295 \\
\hline \multicolumn{4}{|l|}{ Sex } \\
\hline Male & Ref & & \\
\hline Female & 0.734 & -2.313 to 3.781 & 0.637 \\
\hline
\end{tabular}

Abbreviations: EVC, external valve collapse; EVN, external valve narrowing; IVC, internal valve collapse; IVN, internal valve narrowing; NOSE, Nasal Obstruction Symptom Evaluation; VIF, variance inflation factor.

Note: Total septum scores taken as inferior and superior scores found to be collinear. All regression variables with VIF $\leq 3$.

deviation were predictive of higher NOSE scores, and thus increased severity of nasal obstruction. Internal nasal valve narrowing and septal deviation were predictive of symptomatic nasal obstruction for patients with both unilateral and bilateral nasal obstruction.

The use of NOSE scores as a validated patient-reported outcome measure (PROM) has become a standard means of assessing patients with NAO in clinical research. The 2017 Clinical Practice Guide on Rhinoplasty recommends PROMs for clinical use and is supported by the American Academy of Facial Plastic and Reconstructive Surgery and the American Society of Plastic Surgeons. ${ }^{16}$ Although the demographic and anatomic factors that influence NOSE scores have been previously analyzed, the relationship between NOSE scores and specific unilateral nasal physical exam finds has not been previously reported. ${ }^{8}$ In this study all demographic and anatomic variables were first tested with univariate analysis to identify associations, which were then reviewed in multivariate analysis to control for confounding relationships. Multivariate analysis demonstrated that bilateral and unilateral septal deviation and IVN physical exam scores were predictive of elevated NOSE scores. This finding demonstrates the importance of unilateral nasal obstruction even when the opposite side it not obstructed.

Physical exam scales for specific anatomic regions of the nose have previously been developed (turbinoplasty grading and lateral nasal wall insufficiency). ${ }^{12,17}$ The NAW used by the senior author (R.W.L.) incorporates physical exam grading 
scales for multiple areas including the septal deviation, internal nasal valve narrowing, external nasal valve narrowing, and lateral wall insufficiency. ${ }^{9,12,18,19}$ Measurements are reported for both the left and right sides of the nose. For the septal deviation component of the NAW, a Likert scale from 0 to 3 was used so that 0 was used to describe no septal deviation. For the nasal valve narrowing and collapse components, a Likert scale from 1 to 3 was used as previously validated for lateral wall insufficiency. ${ }^{20}$ In 2013, Tsao et al validated a scale for lateral wall insufficiency with grade 1 representing $<33 \%$ collapse, grade 2 representing 33 to $66 \%$ collapse, and grade 3 representing $>66 \%$ collapse. ${ }^{20}$ A previous study demonstrated a composite NAW score combining all 12 parameters was predictive of increased NOSE score on multivariate analysis. ${ }^{8}$ The focus of this study was to utilize the NAW to determine the impact of unilateral nasal obstruction on symptom severity. Through the NAW score, this study uniquely demonstrates the association between specific physical exam finding scores and the severity of NAO.

Understanding the impact of unilateral nasal obstruction will help to inform providers caring for patients with nasal obstruction, highlight to insurance companies that patients with unilateral obstruction require correction, and to assist in the development of clinically meaningful objective outcomes measures. Given this information providers can better communicate with patients about the impact of unilateral nasal obstruction on their personal symptoms to explain the severity of their symptoms despite unilateral obstruction. Understanding the disease-specific and global QoL impact of unilateral nasal obstruction will allow for future health utility evaluations to determine patients who are surgical candidates. Furthermore, as objective measures are developed to directly or indirectly measure nasal airflow and resistance, it is important to understand how to utilize these results to improve the diagnosis and treatment of nasal obstruction. Normal total airflow for a patient with unilateral obstruction may not mean that the patient is asymptomatic if the majority of the nasal airflow is only on one side.

This study has several limitations. Given that the study was performed at a single tertiary academic center with a single surgeon, selection bias may have been introduced where patients may have required higher acuity or complex care and all physical exams were performed by a single surgeon. In addition, patients were only included in the NOSE correlation portion of the study if they completed a preoperative NOSE survey and agreed to have their information used for research purposes. Patients completed the baseline NOSE on the day of initial clinic visit, which may have caused patients to focus on their disease and rate their disease as having a more negative impact on their QOL compared with their average baseline; however, this should be true for patients with unilateral and bilateral nasal obstruction. Despite these limitations, the study has a large sample size of patients and offers valuable insight into the relationship between physical exam findings and symptomatic nasal obstruction.

Future research is needed to understand the reason that bilateral nasal airflow is important for the perception of appropriate nasal airflow. Recent research has discussed the importance of mucosal cooling and sensory feedback to prevent symptoms of nasal obstruction, but unilateral versus bilateral symptoms have not been previously discussed. ${ }^{21}$ As the field of nasal obstruction moves toward the use of objective measures, including computational fluid dynamic models, it is important for clinicians and investigators to understand the clinical importance of unilateral nasal obstruction on a patient's overall perception of nasal airflow.

\section{Conclusion}

Physical exam findings, including septal deviation and IVN, are predictive of an increased NOSE score, regardless of laterality. Unilateral nasal obstruction can cause the same level of symptomatic nasal obstruction as patients with bilateral obstruction. Therefore, patients with unilateral symptoms or physical exam findings should be treated for their symptomatic nasal obstruction despite having only unilateral obstruction.

\section{Conflict of Interest}

None.

\section{References}

1 Chandra RK, Kern RC, Cutler JL, Welch KC, Russell PT. REMODEL larger cohort with long-term outcomes and meta-analysis of standalone balloon dilation studies. Laryngoscope 2016;126 (01):44-50

2 Rhee JS, Poetker DM, Smith TL, Bustillo A, Burzynski M, Davis RE. Nasal valve surgery improves disease-specific quality of life. Laryngoscope 2005;115(03):437-440

3 Gadkaree SK, Fuller JC, Justicz NS, et al. A comparative health utility value analysis of outcomes for patients following septorhinoplasty with previous nasal surgery. JAMA Facial Plast Surg 2019;21(05):402-406

4 Gadkaree SK, Fuller JC, Justicz NS, et al. Health utility values as an outcome measure in patients undergoing functional septorhinoplasty. JAMA Facial Plast Surg 2019;21(05):381-386

5 Gliklich RE, Metson R. The health impact of chronic sinusitis in patients seeking otolaryngologic care. Otolaryngol Head Neck Surg 1995;113(01):104-109

6 Stewart MG, Witsell DL, Smith TL, Weaver EM, Yueh B, Hannley MT. Development and validation of the Nasal Obstruction Symptom Evaluation (NOSE) scale. Otolaryngol Head Neck Surg 2004; 130(02):157-163

7 Lindsay RW. Disease-specific quality of life outcomes in functional rhinoplasty. Laryngoscope 2012;122(07):1480-1488

8 Justicz N, Gadkaree SK, Fuller JC, Locascio JJ, Lindsay RW. Preoperative characteristics of over 1,300 functional septorhinoplasty patients. Laryngoscope 2020;130(01):25-31

9 Colaianni CA, Levesque PA, Lindsay RW. Integrating data collection into office work flow and electronic health records for clinical outcomes research. JAMA Facial Plast Surg 2017;19(06):528-532

10 Harris PA, Taylor R, Thielke R, Payne J, Gonzalez N, Conde JG. Research electronic data capture (REDCap)-a metadata-driven methodology and workflow process for providing translational research informatics support. J Biomed Inform 2009;42(02): 377-381

11 Rhee JS, Sullivan CD, Frank DO, Kimbell JS, Garcia GJ. A systematic review of patient-reported nasal obstruction scores: defining normative and symptomatic ranges in surgical patients. JAMA Facial Plast Surg 2014;16(03):219-225, quiz 232 
12 Most SP. Analysis of outcomes after functional rhinoplasty using a disease-specific quality-of-life instrument. Arch Facial Plast Surg 2006;8(05):306-309

13 Lipan MJ, Most SP. Development of a severity classification system for subjective nasal obstruction. JAMA Facial Plast Surg 2013;15 (05):358-361

14 Miljeteig $\mathrm{H}$, Hoffstein V, Cole P. The effect of unilateral and bilateral nasal obstruction on snoring and sleep apnea. Laryngoscope 1992;102(10):1150-1152

15 Hsu JC, Watari I, Funaki Y, Kokai S, Ono T. Unilateral nasal obstruction induces degeneration of fungiform and circumvallate papillae in rats. J Formos Med Assoc 2018;117(03):220-226

16 Ishii LE, Tollefson TT, Basura GJ, et al. Clinical practice guideline: improving nasal form and function after rhinoplasty executive summary. Otolaryngol Head Neck Surg 2017;156 (02):205-219
17 Camacho M, Zaghi S, Certal V, et al. Inferior turbinate classification system, grades 1 to 4 : development and validation study. Laryngoscope 2015;125(02):296-302

18 Leitzen KP, Brietzke SE, Lindsay RW. Correlation between nasal anatomy and objective obstructive sleep apnea severity. Otolaryngol Head Neck Surg 2014;150(02):325-331

19 Lindsay RW, George R, Herberg ME, Jackson P, Brietzke S. Reliability of a standardized nasal anatomic worksheet and correlation with subjective nasal airway obstruction. JAMA Facial Plast Surg 2016;18(06):449-454

20 Tsao GJ, Fijalkowski N, Most SP. Validation of a grading system for lateral nasal wall insufficiency. Allergy Rhinol (Providence) 2013; 4(02):e66-e68

21 Bailey RS, Casey KP, Pawar SS, Garcia GJ. Correlation of nasal mucosal temperature with subjective nasal patency in healthy individuals. JAMA Facial Plast Surg 2017;19(01):46-52 